\title{
A Surgery on Deep Infiltrating Endometriosis Involving the Rectum: A Debate Started 100 Years Ago between Cullen and Sampson
}

\author{
John L Yovich
}

\begin{abstract}
A recent randomized control trial reports at the 5 years postoperative stage for limited vs extended surgery involving the rectovaginal septum. For those gynecologists with advanced laparoscopy skills who have been reluctant to embrace the idea of complete bowel resections with reanastomosis, the study provides comfort in showing no difference in long-term outcomes between nodule excision and rectal resection. However, the study perpetuates the idea that all medical procedures have to be subjected to this type of statistical analysis, without any reference to the pioneers whose ideas formed the basis of current procedures as well as providing an understanding of the pathogenesis of the underlying disorder. The two gynecologists who first reported on the surgical management of this condition 100 years ago projected different ideas on pathogenesis as well as the appropriate surgical method to apply. Thomas Cullen and John Sampson should be acknowledged in any consideration of determining the appropriate procedure for this challenging disorder.

Keywords: Adenomyosis, Advanced laparoscopic surgery, Bowel resection, Deep infiltrating endometriosis involving rectum DIER, Endometriosis. World Journal of Laparoscopic Surgery (2020): 10.5005/jp-journals-10033-1400
\end{abstract}

\section{INTRODUCTION}

A recently reported randomized controlled study $(\mathrm{RCT})$ regarding surgery for deep infiltrating endometriosis involving the rectum (DIER) showed no significant difference in long-term outcomes between nodule excision vs rectal resection. ${ }^{1}$ The impressive study is reporting at the 5 years postoperative stage but has not actually advanced either the understanding of the underlying disorder nor its proper management. In part, this is due to a failure to consider historical aspects related to the two gynecologists who first reported on this disease, namely, Thomas Stephen Cullen (18681953) and John Albertson Sampson (1873-1946) and who expressed different views on its surgical management 100 years ago.

\section{Thomas Cullen}

Cullen graduated in Medicine in Toronto, Canada, in 1890, thereafter training as a gynecologist at the Johns Hopkins University 1891 but also spending time working as a pathologist in Gottingen, Germany, before returning to Johns Hopkins in 1893. However, the senior surgical position he had expected with Howard Kelly was deferred; hence he established a pathology laboratory, becoming the first Gynecological Pathologist in North America, while also practising as a gynecologist in private practice. He eventually gained the position as Head of Gynecology in 1919 after the retirement of Kelly and had the title of Professor of Clinical Gynecology, a position he held until retirement in 1939. Between 1894 and 1909, Cullen wrote four books on gynecological diseases that married histopathology with clinical symptoms and signs, one of which was adenomyoma of the uterus. ${ }^{2}$ Subsequently, in 1914, he published on the specific subject of adenomyosis of the rectovaginal septum ${ }^{3}$ as well as an accumulated experience in 2020 of his findings of extrauterine adenomyosis, detailing 10 sites that he had personally documented. ${ }^{4}$ However, the worst cases were those involving the rectovaginal septum, numbering 19 in total, and which led to its eponymous title "Cullen's Disease".
PIVET Medical Centre, Perth Western Australia, Australia

Corresponding Author: John L Yovich, PIVET Medical Centre, Perth Western Australia, Australia, Phone: +61419906953, e-mail: jlyovich@ pivet.com.au

How to cite this article: Yovich JL. A Surgery on Deep Infiltrating Endometriosis Involving the Rectum: A Debate Started 100 Years Ago between Cullen and Sampson. World J Lap Surg 2020;13(2):94-96.

Source of support: Nil

Conflict of interest: None

With respect to the specific surgical procedure for DIER, Cullen stated "The removal of an extensive adenomyoma of the rectovaginal septum is infinitely more difficult than a hysterectomy for carcinoma of the cervix". In tackling this disease, Cullen believed that gynecologists should be trained as fully competent abdominal surgeons. ${ }^{3,4}$ He stated: "Where the lumen of the bowel is greatly narrowed, a complete segment of the rectum should be removed with the uterus, and an anastomosis should be made." In such cases "surgeons should perform a "preliminary permanent colostomy... later the pelvic structures can be removed en bloc" . $^{4,5}$

However, despite Cullen's anatomical knowledge and surgical expertise, especially that involving bowel anastomoses, ${ }^{3-5}$ he described some unpleasant complications that included vesicovaginal and rectovaginal fistulas. In this pre-antibiotic era, despite the advanced sterile surgical techniques practiced at the Johns Hopkins Hospital, most of the women who had complications died. $^{4,6}$

\section{John Sampson}

Sampson's experience overlaps with Cullen in that he graduated from Johns Hopkins in 1899 then proceeded into residency in gynecology under Howard Kelly through to 1906, a period when 
Cullen was teaching and provided mentorship. In this period at Johns Hopkins, Sampson published 17 articles and book reviews on various medical disorders, including gynecological subjects as well as surgeries on the pelvic ureter. In 1908, Sampson reviewed Cullen's book on Adenomyomata of the Uterus for Annals of Surgery, a book that undoubtedly captured his imagination and influenced his future research path. Thereafter, Sampson moved into private practice in Albany, New York, and was attached to Albany Medical College where he became Professor of Gynecology through to his retirement in 1945.

At Albany, Sampson developed our current understanding of the condition of endometriosis, including its pathogenesis. In 1913, he described vascular features related to uterine myoma and the unique venous drainage underlying abnormal uterine bleeding. ${ }^{7}$ In 1918 , he demonstrated a metastatic mechanism that can form the basis of a theory for endometriosis in unusual locations. ${ }^{8}$ His betterknown theory of an implantation mechanism following retrograde menstruation was a later idea published in $1927 .^{9}$

Sampson also discussed the clinical management of adenomyosis involving the rectovaginal septum, with viewpoints contrary to those of Cullen. In 1921, he described operations on 23 cases of deep infiltrating rectovaginal endometriosis, concluding "I have never resorted to the extremely radical operations (referring to bowel resections). I have purposely kept close to the uterus, undoubtedly sometimes leaving adenoma in the rectal wall". All these cases had hysterectomy with bilateral salpingooophorectomy (strongly advising not to leave any ovarian tissue in order to ensure that the endometriosis tissue would regress). Sampson stated that "on the whole, the results have been quite satisfactory because the growth is usually only mildly invasive". ${ }^{\prime 9}$

\section{Pathogenesis of Adenomyosis and DIE}

As mentioned earlier, in 1918, Sampson demonstrated a metastatic mechanism $^{8}$ that, I believe, forms the basis of a theory for endometriosis in unusual locations, including the cul-de-sac as an extension from areas of adenomyosis in the posterior uterine wall. ${ }^{9}$ It also explains the mechanism for the formation of adenomyosis within the uterus when the protective "anemic zone" of venules is disrupted. In cases where the uterus is distorted by either being deeply retroverted and retroflexed, particularly if it also included myomata, this predilects to the formation of adenomyosis in the posterior wall. ${ }^{7}$ Sampson described these underlying uterine features in two-thirds of his cases of DIER. His better-known theory of implantation following retrograde menstruation was a later idea published in $1927^{8}$ and which, while explaining the majority of peritoneal endometriosis, does not really explain DIER or endometriosis located in unusual, even extrapelvic sites. The latter are, to my mind, well explained by the metastatic process, and such needs to be understood when undertaking surgical corrections. This means the surgical procedure should include ventrosuspension of the uterus and remove all leiomyomata along with resection of adenomyoma in addition to en bloc excision of the rectovaginal nodules.

\section{Nodular Excision vs Bowel Resection for DIER}

Having adopted laparoscopy in 1973, I attended the facility in Clermont-Ferrand, France, headed by Maurice Bruhat in the early
1980 s to advance my skills. His vision included that gynecologists who would manage women with endometriosis should develop their laparoscopic skills to an advanced level and also be competent in the broader context of abdominal surgery in order to manage colorectal and urogenital aspects, as these areas were often involved. ${ }^{10}$ Some of Bruhat's protégés have reached very high levels of expertise and undertake segmental bowel resections using linear and circular stapling devices. ${ }^{11}$ However, I, along with most gynecologists who have advanced laparoscopy skills, shy away from resections, preferring to follow the advice of those like Jacques Donnez ${ }^{12}$ that "at the level of the bowel, a ring of fibrosis may be left behind" without any future concern. The decision of which approach to follow must undoubtedly depend upon case numbers managed, as using the stapling device requires a practiced skill. Given that my group has recognized only 30 such cases over a 30 years period involving laparoscopic excisional surgery for around 4,000 cases of endometriosis and adenomyosis, the idea of developing competency with bowel-resection devices was simply too foreboding. ${ }^{9}$

\section{Conclusion}

This commentary is presented in response to the recent RCT study reported from France which applied advanced statistics to evaluate the surgical management of DIER - either by colorectal resection/re-anastomosis or by a more conservative surgical procedure involving nodular resection. The clinical outcomes were similar, undoubdtedly because the surgeons were each utilizing the procedure with which they were comfortable. Of course, I am very pleased that my conservative surgery was not found to be statistically inferior from those who undertook bowel resections, but I remain in admiration of their ability to avoid complications.

As a laparoscopic surgeon with 47 years of experience, I am also perplexed at the perceived need to subject every medical process and surgical procedure to an RCT while completely ignoring the historical evolution of the subject. With respect to the sister conditions of endometriosis and adenomyosis, I have always turned to John Sampson for the best advice; his descriptive articles from a century ago have been, for me, fully clarifying.

\section{ACKnOWLedgments}

I thank those colleagues who assisted me to establish advanced laparoscopic skills for treating the infertility-related conditions of endometriosis, adenomyosis and leiomyomata. Their patience and support have also enabled them to develop the necessary advanced skills for the preparation of young women undergoing fertility-related management. This was achieved by surrounding the patient with three gynecologists at different stages of career development (operator, assistant, and bottom-end manipulator) for advanced surgeries. This meant income opportunities were diluted but their names are reflected in our collaborative publications over these 40 years.

\section{References}

1. Roman $\mathrm{H}$, Tuech JJ, Huet $\mathrm{E}$, et al. Excision vs colorectal resection in deep endometriosis infiltrating the rectum: 5 year follow-up of patients enrolled in a randomised controlled trial. Hum Reprod 2019;34(12):2362-2371. DOI: 10.1093/humrep/dez217.

2. Cullen TS. Adenomyoma of the Uterus. Philadelphia: W. B. Saunders; 1908. p. 294. 
3. Cullen TS. Adenomyoma of the rectovaginal septum. JAMA 1914;62:835-839. DOI: 10.1001/jama.1914.02560360015006.

4. Cullen TS. Adenoma of the recto-vaginal septum. JAMA 1916;67: 401-406. DOI: 10.1001/jama.1916.02590060001001.

5. Cullen TS. The distribution of adenomyomas containing uterine mucosa. Arch Surg 1920;1(2):215-283. DOI: 10.1001/ archsurg.1920.01110020002001.

6. Yovich JL. The history of endometriosis preceding Sampson. Med J Obstet Gynecol 2020;8(1):1131, 1-8.

7. Yovich JL, Rowlands PK, Lingham S, et al. Advanced fibroid study: paying homage to john Sampson. Reprod Biomed Online 2019;39(2):183-186. DOI: 10.1016/j.rbmo.2019.04.013.

8. Yovich JL, Rowlands PK, Lingham S, et al. Pathogenesis of endometriosis: look no further than john Sampson. Reprod
Biomed Online 2020;40(1):7-11. DOI: 10.1016/j.rbmo.2019 10.007.

9. Yovich JL. Understanding endometriosis: clarifying Sampson's theories with a personal perspective. Med J Obstet Gynecol 2020;8(1):1130, 1-12.

10. Canis M, Mage $G$, Manhes $H$, et al. Laparoscopic treatment of endometriosis. Acta Obstet Gynecol Scand 1989;68(S150):15-20. DOI: 10.1111/aogs.1989.68.s150.15.

11. Afors K, Centini G, Fernandes R, et al. Segmental and discoid resection are preferential to bowel shaving for medium-term symptomatic relief in patients with bowel endometriosis. JMIG 2016;23(7): 1123-1129. DOI: 10.1016/j.jmig.2016.08.813.

12. Donnez J. Endometriosis: enigmatic in the pathogenesis and controversial in its therapy. Fertil Steril 2012;98(3):509-510. DOI: 10.1016/j.fertnstert.2012.07.1125. 